\title{
Desarrollo de la complejidad sintáctica en recontados narrativos de niños preescolares y escolares ${ }^{1}$
}

\author{
Development of Syntactic Complexity in Narrative Retellings by Preschool \\ and School-Age Children
}

Recibido: Junio 2016 Aceptado: Julio 2017 Publicado: Noviembre 2017

\begin{tabular}{|c|c|c|}
\hline Christian Peñaloza & Claudia Araya & Carmen Julia Coloma \\
\hline $\begin{array}{l}\text { Universidad de Chile } \\
\text { Chile }\end{array}$ & $\begin{array}{l}\text { Universidad de Chile } \\
\text { Chile }\end{array}$ & $\begin{array}{l}\text { Universidad de Chile } \\
\text { Chile }\end{array}$ \\
\hline cpenaloza@med.uchile.cl & carayac@med.uchile.cl & ccoloma@med.uchile.cl \\
\hline
\end{tabular}

Resumen: El aumento de la complejidad sintáctica evidencia el desarrollo lingüístico de los niños. El uso de ciertas medidas de lenguaje permite dar cuenta de dicho proceso, lo que ha sido corroborado en niños preescolares hispanohablantes y en niños en etapas tardías de desarrollo. Este trabajo propone explorar dicho desarrollo contrastando a un grupo de niños chilenos con desarrollo típico que asisten a preescolar con un grupo de primer año básico. Las medidas incluyeron índices primarios y secundarios de complejidad y se obtuvieron a partir de recontados narrativos producidos por los niños. En los resultados se observa que no todos los índices aumentan al contrastar los dos grupos. Solo aumenta un índice primario de densidad clausular dentro de las oraciones complejas y el número de subordinadas sustantivas, particularmente en las etapas narrativas de presentación y final; en paralelo, disminuye el número de subordinadas adverbiales en la fase de final. Estos resultados evidencian que, si bien se observa un aumento en algunas medidas de complejidad al contrastar los grupos, el desarrollo de estas habilidades no presenta un mismo patrón en todas las dimensiones, lo que puede deberse a factores discursivos o del propio proceso de desarrollo lingüístico.

Palabras clave: medidas de complejidad sintáctica - niños hispanohablantes recontados narrativos

Citación: Peñaloza, C., Araya, C. \& Coloma, C. J. (2017). Desarrollo de la complejidad sintáctica en recontados narrativos de niños preescolares y escolares. Logos: Revista de Lingüística, Filosofía y Literatura 27(2), 333-348. DOI: 10.15443/RL2726

Dirección Postal: Avda. Independencia 1027, Independencia, Santiago, Chile 


\begin{abstract}
The increase of syntactic complexity evidences the linguistic development of children. The use of certain language measures makes it possible to account for this process, which has been corroborated in Spanish-speaking preschool children and children in late stages of language development. This work proposes to explore this development by contrasting a group of Chilean children with typical development who attend preschool with a group of first grade school children. The measures included primary and secondary indices of complexity, and were obtained from narrative retellings produced by the children. Results show that not all the indices increase when the two groups are compared. Only a primary index of clausular density within the complex sentences increases and the number of noun subordinates, particularly in the narrative stages of presentation and ending; in parallel, the number of adverbial subordinates in the final phase decreases. These results show that, although there is an increase in some measures of complexity in contrasting groups, the development of these skills does not present a constant rhythm in all dimensions, which may be due to discursive factors or to the process of linguistic development itself.
\end{abstract}

Keywords: Measures of syntactic complexity - ungrammaticality - Spanishspeaking children - narrative retellings

\title{
1. Introducción
}

El desarrollo de la complejidad sintáctica es un fenómeno de suma importancia en el aprendizaje lingüístico de los niños. Este concepto corresponde a la capacidad de relacionar dos o más unidades predicativas verbales bajo parámetros de gramaticalidad y coherencia, lo que da cuenta de la habilidad del niño para ajustar el contenido y la forma a las necesidades comunicativas que surjan en su interacción con su interlocutor (Auza \& Alarcón, 2011). En niños con desarrollo típico se ha observado que esta capacidad crece y se diversifica a mayor edad (Castilla \& Eriks, 2011; Coloma, Peñaloza \& Fernández, 2007), lo que parece evidenciar de buena manera el desarrollo lingüístico general de los niños (Hunt, 1970; Véliz, 1988). Además, la complejidad sintáctica se ha relacionado con el desempeño lector de niños tanto con desarrollo típico como con problemas lingüísticos (Storch \& Whitehurst, 2002; Bishop \& Adams, 1990). En niños hispanohablantes, la sintaxis compleja suele aparecer en torno a los 30 meses de edad, aunque la productividad de las estructuras y funciones involucradas no se consolida sino hasta después de los tres años (Serra, Serrat, Solé, Bel \& Aparici, 2000). Durante todo el período preescolar, los niños aumentan y diversifican su producción de cláusulas subordinadas, de tal manera que los principales tipos se encuentran presentes a los seis años, aunque algunas aparezcan en bajo número o no se tenga certeza de que su uso dé cuenta de la comprensión cabal de las relaciones lógicas que expresa, en el caso de algunas adverbiales. La escolaridad, a su vez, plantea nuevos desafíos comunicativos a los niños, lo que impacta en que desarrollen nuevas formas complejas, tales como subordinadas adjetivas con nexos propios de la escritura, o la consolidación de subordinadas finales y concesivas, que requieren el dominio del subjuntivo (Serra et al., 2000). En paralelo, existen indicios de que algunos aspectos de la complejidad se presentan disminuidos o alterados en niños con dificultades en su aprendizaje lingüístico, por lo que podrían ser un buen discriminador entre niños con y sin desarrollo típico del lenguaje (Castilla \& Eriks, 
2011; Jackson \& Maldonado, 2015; Pavez, Coloma, Araya, Maggiolo \& Peñaloza, 2015; SimónCereijido \& Gutiérrez-Clellen, 2007).

En la tradición hispanista, el concepto de sintaxis compleja ha sido utilizado para describir aquellas construcciones oracionales en que uno o más verbos se subordinan a un verbo principal (Gili Gaya, 1948; Real Academia Española, 2010). La oración así conformada recibe el nombre de oración compleja, la cual presenta un verbo principal y uno o más subordinados. Cada verbo subordinado, junto a sus constituyentes, se denominan oraciones subordinadas, y suelen recibir nombres según su función dentro de la predicación principal y su forma. Así, se suelen reconocer tres grandes tipos de subordinadas: las de relativo, las nominales y las llamadas adverbiales, etiqueta que reúne una diversa gama de construcciones subordinadas, algunas propiamente adverbiales, como las locativas, y otras sin equivalente funcional adverbial, como las concesivas (Real Academia Española, 2010).

Se pueden reconocer, sin embargo, concepciones más o menos similares en otras tradiciones lingüísticas. De gran importancia son las propuestas desarrolladas tanto por el distribucionalismo como por el generativismo estadounidense, que surgen de principios teóricos distintos, pero que dieron lugar a operacionalizaciones llamadas medidas de complejidad. En el caso del distribucionalismo, la noción de complejidad se relaciona, grosso modo, con el número de palabras o morfemas que se observa en una unidad lingüística; en el segundo modelo, con el número de transformaciones en la estructura profunda que se suponen en la conformación de dicha unidad. Una revisión teórica al respecto se puede encontrar en Checa (2003) y en Crespo, Alfaro y Góngora (2011).

Dadas las diversas concepciones que existen al respecto, dar cuenta de la sintaxis compleja dentro del desarrollo infantil se ha vuelto un constante desafío para los investigadores y se ha abordado desde diferentes perspectivas. Por ejemplo, se ha estudiado en relación al uso de nexos subordinantes bajo el supuesto de que su análisis da cuenta de la diversidad funcional y estructural de las unidades complejas que producen los niños (Alarcón \& Auza, 2011). Otras investigaciones se enfocan en describir la diversidad funcional y estructural en que aparecen los verbos subordinados en las producciones infantiles (Coloma et al. 2007; Gili Gaya, 1972; Jackson \& Maldonado, 2015; Peñaloza, 2008). En estos casos, la principal disyuntiva es si la descripción se basa en la mera aparición de un recurso lingüístico (por ejemplo, la subordinada de infinitivo con función de objeto directo) o si se opta por algún criterio que asegure que la unidad en cuestión es productiva y está consolidada en el lenguaje, lo que resulta muy relevante en estudios de edades tempranas de desarrollo (Serra et al., 2000; Rojas, 2009). En este sentido, han tenido una particular importancia los trabajos sobre la aparición y consolidación de las subordinadas de relativo en niños hispanohablantes (Silva \& Plana, 2014).

Uno de los métodos más fructífero para dar cuenta del desarrollo de la complejidad sintáctica ha sido la operacionalización del fenómeno a través de las ya mencionadas medidas de lenguaje. Estas son cuantificaciones de ciertos aspectos léxicos y sintácticos que permiten realizar comparaciones entre grupos de distintas edades, orígenes o grados de desarrollo lingüístico (Castilla \& Eriks, 2011). Muchas de estas medidas se basan en la noción de complejidad, por lo que son el método usado por muchos trabajos que abordan este fenómeno.

En estudios con niños angloparlantes, la observación de la complejidad a través de estas medidas es de larga data, particularmente en textos escritos de escolares (Hunt, 1965; McCarthy, 1954). Probablemente, el índice más conocido y utilizado es el de la longitud media, definido como el número de morfemas o palabras por la unidad que se defina, ya sea oración, cláusula o enunciado. Esta medida, según se ha visto, se relaciona con la edad y, por lo tanto, sistemáticamente da cuenta de un desarrollo en la madurez sintáctica (Checa, 2005). En las décadas de los sesenta y setenta se proponen los métodos esenciales de medición vigentes aún hoy, que se distinguen especialmente por la unidad en que se enmarca el análisis: las unidades terminales (unidades-t) y las cláusulas, en el caso de Hunt $(1965,1970)$, aplicado inicialmente al lenguaje escolar escrito; y el enunciado, en el caso de Brown (1973), para el lenguaje de niños 
en etapas tempranas de desarrollo.

En estudios con niños hispanohablantes, ha sido de particular importancia el índice de cláusulas por unidad sintáctica, ya sea unidades-t u oraciones. Esta medida, conocida como índice de complejidad, expresa numéricamente cuántas unidades verbales hay dentro de la unidad sintáctica mayor, lo que indirectamente informa de cuántas cláusulas aparecen subordinadas (Jackson \& Maldonado, 2015). En este trabajo se hará referencia a este índice como COMP-1. Una medida centrada en la subordinación de manera más explícita es el índice de cláusulas subordinadas por oración compleja (Jackson \& Maldonado, 2015; Peñaloza, 2008); es decir, un índice de densidad de subordinadas. Acá se hará referencia a este índice como COMP-2.

En un estudio con niños colombianos con desarrollo típico de tres, cuatro y cinco años, se observó que dichos índices permitían diferenciar las producciones narrativas según la edad y que ambas medidas presentaban una alta correlación entre ellas; es decir, que a mayor edad, los niños emiten unidades más largas y con mayor número de cláusulas (Castilla \& Eriks, 2011). En niños bilingües en Estados Unidos, la longitud media resultó ser un buen predictor de las habilidades lingüísticas medidas a través de una prueba formal, solo cuando se obtenía de recontados en inglés, no así en español (Bedore, Peña, Gillam \& Ho, 2010).

Cualquier índice general de complejidad, tal como los descritos anteriormente, puede complementarse con medidas más específicas, llamadas secundarias clausales, que informan del número de subordinadas adjetivas, sustantivas y adverbiales en la producción infantil (Checa, 2005). Estos índices secundarios surgen a partir de la pregunta de si todos los tipos de subordinadas aumentan su número de igual manera en que lo hace el índice general de subordinación. En inglés, se ha visto que el índice de subordinadas adjetivas por unidad-t se relaciona con la edad de los niños, tanto en etapa preescolar como escolar, lo que no se observa con igual claridad con las subordinadas sustantivas y adverbiales (Hunt, 1965; Loban 1970). En español, Coloma et al. (2007) observaron que los niños de diez años producían un mayor número de subordinadas adjetivas y sustantivas que niños de ocho años, en recontados narrativos sin apoyo de imágenes. El aumento en el número de adjetivas ha sido corroborado por otros estudios con niños de edades similares o mayores (Gutiérrez-Clellen \& Hofstetter, 1994; Muñoz, Echeverría \& Véliz, 1985; Véliz, 1988); lo que no ocurre de igual forma con el número de subordinadas sustantivas, pues este resulta ser muy sensible a si se consideran o no en el conteo fenómenos como el discurso directo referido. Las diferencias también pueden originarse en las distintas modalidades discursivas utilizadas.

Muchas de las investigaciones citadas han trabajado con estos índices para las mismas edades de niños; sin embargo, se observa una alta discrepancia entre sus resultados. Estas diferencias pueden deberse a diversos motivos. En primer lugar, resulta relevante conocer si solo se consideraron como cláusulas las construcciones con verbos finitos, o se incluyeron los infinitivos y gerundios. De igual manera, resulta clave la definición sobre qué elementos se identifican como perífrasis verbales y qué estatus sintáctico se le da al discurso directo referido, dado que los niños emiten este tipo de discurso referido con mucha frecuencia (Jackson \& Maldonado, 2015).

También resulta de importancia el tipo de discurso en que se mide la producción lingüística. Se sabe que, en niños con trastorno del lenguaje, la producción de discursos narrativos genera un mayor índice de complejidad que otros discursos, como conversaciones, interacciones a través de juegos o emisiones argumentativas (Pavez, et al., 2015; Thordardottir, 2008); a su vez, las tareas de recontado generan un índice más alto de complejidad que las tareas de narración espontánea (Auza \& Alarcón, 2011). Por último, se observan patrones distintos no solo según la modalidad (oralidad versus escritura) o el tipo de discurso en que se elicita la producción infantil (narración versus conversación), sino también en las distintas fases dentro de la secuencia textual. De hecho, es esperable que seleccione un conjunto más o menos diferenciado de recursos sintácticos, entre ellos distintos tipos de subordinadas, según los fines retóricos y de significado que se plasman en cada fase de una secuencia discursiva (Benítez \& Alvarado, 
2013). En relación a lo anterior, Peñaloza (2008) observó que los porcentajes de aparición de subordinadas adjetivas, sustantivas y adverbiales resultaron no ser iguales en las distintas fases de recontados narrativos de niños de seis, ocho y diez años, y que dichas proporciones se mantenían relativamente estables en las distintas edades. Meneses, Ow \& Benítez (2012) establecieron que, en producciones escritas y orales de escolares, las fases secuenciales de sus textos expositivos y narrativos se caracterizaban por alcanzar distintos índices de complejidad. Se observa así que, en el recontado narrativo, pareciera manifestarse de manera más clara que en otras secuencias y modalidades el desarrollo de la complejidad. Estas observaciones se han realizado principalmente en producciones narrativas de niños en etapa escolar, por lo que resulta relevante verificar si estos rasgos ya están presentes en el discurso de niños preescolares, que aún están en etapa de consolidación de sus habilidades narrativas.

En síntesis, los niños que transitan de la etapa preescolar a la escolar están en pleno desarrollo de sus habilidades gramaticales, lo que incluye la complejidad sintáctica. El uso de ciertas medidas de lenguaje permite dar cuenta de dicho proceso; aunque por lo general estas medidas se han considerado por separado y en períodos de desarrollo tardío. Dado que aún carecemos de información relevante en torno al desarrollo de la complejidad sintáctica en niños hispanohablantes chilenos entre el período preescolar y escolar, se propone aportar con información a partir de los datos observados en una misma muestra de lenguaje, en este caso narrativo. El objetivo de este trabajo, entonces, es dar cuenta del desarrollo de la complejidad sintáctica en la producción narrativa de niños de primer año básico en contraste con niños en etapa preescolar, por medio de una serie de medidas de complejidad. La hipótesis de trabajo es que las producciones del grupo de escolares presentarán mayor complejidad sintáctica, tanto en los índices principales como secundarios, de manera global y en cada fase narrativa. Esta información permite conocer algunos elementos que conforman el patrón de desarrollo lingüístico de niños chilenos, especialmente en una etapa tan crítica como es el término del periodo preescolar y el ingreso a la escolaridad.

\section{Método y Materiales}

\subsection{Criterios de selección}

Grupo en estudio 1 (G1): a) presentar desarrollo típico de lenguaje, informado por la fonoaudióloga del establecimiento educacional, b) evidenciar audición y habilidades cognitivas no verbales dentro de rangos normales, c) ser alumnos preescolares de escuelas municipales o particulares subvencionadas.

Grupo en estudio 2 (G2): a) presentar desarrollo típico de lenguaje, informado por la fonoaudióloga de la escuela, b) evidenciar audición y habilidades cognitivas no verbales dentro de rangos normales, c) ser alumnos de $1^{\circ}$ básico de escuelas municipales o particulares subvencionadas.

\subsection{Características de los participantes}

Los participantes pertenecen a establecimientos educacionales municipales y particulares subvencionados con Proyectos de Integración (PIE), es decir, colegios regulares que incorporan a sus aulas a niños con necesidades especiales, con apoyo de fonoaudiólogos/as.

Se seleccionaron 40 niños distribuidos en los dos grupos mencionados. El G1 estaba constituido por 20 pre-escolares (10 niños y 10 niñas) que tenían un promedio de edad de 4.4 años. El G2 estaba conformado por 20 escolares (12 niños y 8 niñas) con una edad promedio de 6.6 años.

\subsection{Instrumentos utilizados en la selección de los participantes}

El desempeño auditivo se midió a través de una audiometría de barrido en las frecuencias 500, 1000, 2000 y $4000 \mathrm{hz}$. Se utilizaron los criterios internacionales propuestos por la ASHA que determinan la normalidad auditiva a intensidades iguales o inferiores a $20 \mathrm{~dB}$ (American Speech 
-Language-Hearing Association, 2005).

Las habilidades cognitivas, por su parte, se valoraron a través del Test de Matrices Coloreadas y Progresivas de Raven, que implica tareas de razonamiento por analogías visuales mediante la comparación de figuras. Según el test, las puntuaciones ubicadas en el percentil 25 o sobre este se interpretan como normales (Raven, 2005).

Todos los niños presentaron audición y habilidades cognitivas dentro de los rangos normales para sus edades.

\subsection{Procedimientos de evaluación}

Se evaluó individualmente a los niños en dos sesiones: en la primera se aplicó la audiometría y se midieron las habilidades cognitivas no verbales. En la segunda se recogió la muestra de lenguaje, que consistió en una producción narrativa inducida por guiones, los cuales fueron recontados por cada niño. Esta estrategia de elicitación permite que los niños emitan un mayor número y diversidad de oraciones complejas (Auza \& Alarcón, 2011).

Los cuentos con que se trabajó fueron "La ardillita glotona", "El sapito saltarín" y "El lobo friolento" (Pavez, Coloma \& Maggiolo, 2008). Estos cuentos han sido utilizados para valorar el desarrollo de las habilidades narrativas en niños chilenos con desarrollo típico y con trastorno específico del lenguaje (TEL). Son relatos establecidos en guiones que presentan una complejidad narrativa creciente, según ciertas características de su estructura narrativa, y que presentan las siguientes características gramaticales: en conjunto, presentan 56 oraciones subordinadas, de las cuales ocho son adjetivas; veinticuatro, sustantivas, y veinticuatro, adverbiales. Los tres guiones presentan subordinadas de los tres tipos. La fase de presentación de personajes y del conflicto está conformada por un mayor número de subordinadas adjetivas que sustantivas y adverbiales (seis, cuatro y dos, respectivamente); por el contrario, en los episodios, aparecen más adverbiales que adjetivas y sustantivas (diecinueve, una y trece, respectivamente); por último, en la fase de final, los guiones cuentan con más subordinadas sustantivas y adverbiales que adjetivas (seis, cuatro y una, respectivamente).

A todos los niños se les presentan los cuentos en el mismo orden mencionado anteriormente. El procedimiento consiste en que el evaluador lee el guion del primer cuento, sin apoyo de imágenes, y luego se le solicita al niño que lo cuente con sus propias palabras. En caso de que el niño no relate, se le incita a hacerlo con el apoyo “¿Y qué más?”, que se formula con un máximo de tres veces. Luego se procede de la misma manera con el segundo y el tercer cuento.

\subsection{Análisis de la estructura narrativa}

La estructura se analizó identificando tres categorías: presentación, episodio y final. La presentación incluye al personaje y al problema del relato. El espacio y el atributo del personaje son elementos opcionales. El episodio se conforma por una secuencia de acción + obstáculo + resultado; en él también puede aparecer la meta que es optativa. La categoría de final es la información que resuelve el problema de la presentación. La presentación y el episodio pueden ser completos o incompletos. La presentación es incompleta cuando solo se menciona el personaje o el problema. En el caso del episodio, es incompleto si la secuencia es acción + obstáculo u obstáculo + resultado. Se dice que un niño estructura su narración sí ya presenta las categorías de presentación y episodios, aunque estén ambos incompletos.

\subsection{Análisis de las oraciones}

El análisis gramatical de las muestras de lenguaje implicó varios tipos de análisis y conteos. Se segmentaron los tres recontados de cada niño en oraciones (equivalentes a unidades- $t$ ) y se clasificó cada una de ellas en oraciones simples o complejas. En el caso de las complejas, se identificó, a su vez, el número de cláusulas subordinadas que cada una presentaba y estas 
se clasificaron en adjetivas, sustantivas y adverbiales. Los conteos descritos se realizaron, además, en cada fase de la estructura narrativa (presentación, episodios y final). Se identificó como oración simple la unidad mínima de predicación con un verbo conjugado independiente, sin otros verbos (es decir, cláusulas) que lo determinen sintácticamente. Se identificó como oración compleja la presencia de un verbo finito (principal) modificado por uno o más verbos (cláusulas subordinadas). En la identificación de estos últimos, se contabilizó tanto verbos conjugados como infinitivos y gerundios.

Las cláusulas subordinadas adjetivas se identificaron en su función de modificadores directos de un sustantivo explícito, de tal modo que no todas las cláusulas de relativo fueron identificadas como adjetivas. Por su parte, se clasificaron como sustantivas aquellas cláusulas que cumplían una función nominal, como sujeto u objeto del verbo principal. Los enunciados directos con contenido verbal se consideraron objeto del verbo de decir correspondiente y, por lo tanto, se contabilizaron como subordinadas sustantivas. La construcción querer + infinitivo no se consideró perifrástica; es decir, se contabilizó cada infinitivo como cláusula subordinada. Finalmente, se clasificaron como adverbiales las cláusulas con función adverbial (por ejemplo, de lugar) y con otros valores que también han sido considerados adverbiales o circunstanciales (de finalidad, causales, concesivas, etc.). Las estructuras con valor de finalidad para + infinitivo y para + que se consideraron ambas adverbiales.

A partir de estos análisis y conteos, se obtuvieron las distintas medidas lingüísticas que se contrastaron entre los dos grupos de edad. Estas medidas fueron:

1) Índice de cláusulas por oración (COMP-1).

2) Índice de cláusulas subordinadas por oración compleja (COMP-2).

Ambos casos corresponden a los índices primarios, y los valores entregados son iguales o mayores a 1. En el caso del COMP-1, el valor 1 representa que existe un número equivalente de cláusulas y oraciones, es decir que el niño solo emitió oraciones simples; por el contrario, valores que se alejen de 1 expresan una mayor cantidad de cláusulas subordinadas y que, por lo tanto, hubo oraciones complejas. En el segundo índice, el valor 1 señala que en cada oración compleja existió solo una cláusula subordinada; en cambio, valores que se alejen de 1 informan que los niños produjeron, en una misma oración compleja, un mayor número de subordinaciones.

3) Índice de subordinadas adjetivas por oración compleja (COMP-2 Adj).

4) Índice de subordinadas sustantivas por oración compleja (COMP-2 Sust).

5) Índice de subordinadas adverbiales por oración compleja (COMP-2 Adv).

Estos son índices secundarios y los valores entregados son iguales o mayores a cero. Un índice cero indica que el niño no produjo el tipo de subordinada en cuestión en su recontado. Un índice con valor 1 informa que el niño emitió en promedio una subordinada adjetiva, sustantiva o adverbial por oración compleja. Valores sobre 1 indican que el niño tendió a emitir más de una subordinada por oración compleja.

\subsection{Análisis de los datos}

Se contrastaron los datos de los dos grupos de estudios (G1 = preescolares; G2 = escolares de primero básico]. El contraste se realizó a través de una comparación de medias, para lo cual se usó la prueba $t$ de Student de una cola. En todos los casos, se optó por una significancia estadística para valores de $p$ iguales o menores a 0,05.

\section{Resultados}

En primer lugar, se presentan algunos datos descriptivos en que se enmarcan los resultados del estudio. En relación al nivel de desarrollo narrativo, todos los niños de la muestra estructuran sus relatos con presentación y episodio. Once niños de preescolar emitieron el final del cuento, lo que contrasta con los dieciocho niños de primer año que también lo hicieron. Se aplicó una prueba de chi cuadrado para verificar si estas distintas proporciones diferencian a los 
grupos, resultando positiva (chi cuadrado: 6,14; significancia de ,013). Así, todos los niños se encuentran dentro de un nivel de desarrollo narrativo esperado para su edad, aunque los grupos se diferencian por la producción del final. Además, todos emitieron cláusulas subordinadas en sus recontados. El mínimo de subordinaciones producidas por un niño fue de 2 y el máximo, de 19. A continuación se presentan los valores de producción gramatical de ambos grupos (ver Tabla 1).

\begin{tabular}{|l|c|c|c|c|c|c|c|c|}
\hline & \multicolumn{5}{|c|}{ Grupo 1 } & \multicolumn{4}{c|}{ Grupo 2 } \\
\hline & $\mathrm{x}$ & $\mathrm{D} . \mathrm{E}$. & $\mathrm{Min}$ & $\mathrm{Max}$ & $\mathrm{x}$ & $\mathrm{D} . \mathrm{E}$. & $\mathrm{Min}$ & $\mathrm{Max}$ \\
\hline Oraciones & 26,45 & 8,37 & 7 & 45 & 28,9 & 7,4 & 14 & 41 \\
\hline Cláusulas & 34,8 & 11,42 & 9 & 57 & 39,8 & 10,09 & 21 & 60 \\
\hline Oraciones simples & 18,6 & 7,16 & 5 & 34 & 19,55 & 6,42 & 6 & 32 \\
\hline $\begin{array}{l}\text { Oraciones } \\
\text { complejas }\end{array}$ & 7,85 & 4,39 & 2 & 18 & 9,35 & 3,54 & 2 & 17 \\
\hline $\begin{array}{l}\text { Cláusulas } \\
\text { subordinadas }\end{array}$ & 8,35 & 4,62 & 2 & 18 & 10,9 & 4,09 & 2 & 19 \\
\hline & & & & & & & & \\
\hline
\end{tabular}

Tabla 1. Promedios, D.E., valores mínimo y máximo por grupo de la producción verbal.

Como se observa en la Tabla 1, en todos los aspectos cuantificados el grupo de escolares producen un mayor número de unidades que el grupo de preescolar. Se aplicó, una $t$ de Student para observar si en algunas de estas medidas existía diferencias por sexo delo niños, lo que resultó negativo.

A partir de estos valores descriptivos, se obtuvieron los diversos índices que permiten dar cuenta del desarrollo de la complejidad sintáctica en los recontados de estos niños. A continuación se presentan los resultados de dichos análisis, organizados en dos etapas. En primer lugar, los índices principales y secundarios a partir del análisis global de los recontados; posteriormente, los índices principales y secundarios según las distintas categorías o fases de la estructura narrativa.

Índices primarios y secundarios globales

A continuación se presentan los resultados en torno a las dos medidas primarias de complejidad sintáctica global contempladas en este estudio: COMP-1 y COMP-2. A ambos índices se les aplicó una t de Student para verificar si existían diferencias significativas entre los grupos. Los resultados se muestran en la Tabla 2:

\begin{tabular}{|l|c|c|c|c|c|c|}
\hline & \multicolumn{2}{|c|}{ Grupo 1 } & \multicolumn{2}{c|}{ Grupo 2 } & & \\
\hline & $\mathrm{x}$ & D.S. & $\mathrm{x}$ & D.S. & $t(p)$ & $r$ \\
\hline COMP-1 & 1,31 &, 15 & 1,38 &, 13 & $-1,5(, 070)$ & 0,24 \\
\hline COMP-2 & 1,06 &, 12 & 1,16 &, 12 & $-2,6(, 006)^{*}$ & 0,40 \\
\hline
\end{tabular}

x: promedio. D.E.: desviación estándar. t: valor de t en el contraste de medias. p: significancia. r: tamaño del efecto.

$* \mathrm{p}<.05$

Tabla 2. Comparación de los promedios de cláusulas por oración (COMP-1) y de cláusulas subordinadas por oración compleja (COMP-2).

En la Tabla 2 se observa que ambos índices son mayores en la producción narrativa de los 
niños de primero básico en comparación con los niños pre-escolares; sin embargo, el índice de complejidad más comúnmente reportado, el que se obtiene al dividir el total de cláusulas por el total de oraciones, no diferenció significativamente a los grupos de esta muestra; por el contrario, el segundo índice, que informa de la cantidad de subordinaciones dentro de las oraciones complejas, sí presentó diferencias significativas al comparar a preescolares con escolares, aunque con un tamaño de efecto pequeño.

Dado que se observa un aumento significativo en el número de cláusulas subordinadas por oración compleja, se verificará a continuación si esos resultados se replican en cada uno de los tipos de subordinadas (adjetivas, sustantivas y adverbiales). Para esto, se obtuvo el índice de cada tipo de subordinada por oración compleja (COMP-2 Adj, COMP-2 Sust y COMP-2 Adv) y a dichos valores se aplicó la t de Student . Los resultados se muestran en la Tabla 3.

\begin{tabular}{|l|c|c|c|c|c|c|}
\hline & \multicolumn{2}{|c|}{ Grupo 1 } & Grupo 2 & & \\
\hline & $\mathrm{x}$ & D.E. & $\mathrm{x}$ & D.E. & $\boldsymbol{t}(\boldsymbol{p})$ & $\boldsymbol{r}$ \\
\hline COMP-2 Adj & 0,20 & 0,18 & 0,14 & 0,17 & $0,98(, 17)$ & 0,16 \\
\hline COMP-2 Sust & 0,29 & 0,19 & 0,49 & 0,18 & $-3,3(, 001)^{*}$ & 0,48 \\
\hline COMP-2 Adv & 0,56 & 0,25 & 0,50 & 0,21 & $0,87(, 19)$ & 0,14 \\
\hline $\begin{array}{l}\text { x: promedio. D.E.: desviación estándar. t: valor de t en el contraste de medias. p: significancia. r: tamaño } \\
\text { del efecto. } \\
\text { *p }<.05\end{array}$
\end{tabular}

Tabla 3. Comparación de los promedios de cada tipo de cláusulas por oración compleja.

Según los datos de la Tabla 3, se observa que las subordinadas adverbiales son las que presentan una mayor presencia por oración compleja en el grupo 1, seguidas por las sustantivas; sin embargo, solo estas últimas aumentan su presencia al contrastar los dos grupos, con un incremento, además, estadísticamente significativo y un tamaño de efecto mediano. En cuanto al grupo 2, se advierte que las adverbiales y las sustantivas tienen un promedio similar y las adjetivas, al igual que en el grupo 1 , son las que menos se producen.

Índices primarios y secundarios por fase narrativa

Hasta acá, se observa que las producciones gramaticales de niños de preescolar y de primero básico, en una tarea de recontado narrativo, se diferencian en el número de cláusulas subordinadas por oración compleja, manifestado particularmente en un aumento significativo en la producción de subordinadas sustantivas. A continuación se presentan los resultados al contrastar los distintos índices según las fases de la secuencia narrativa en que se producen. Para esto, se observará cómo se comportan los índices principales y secundarios en cada una de las categorías (presentación, episodios, final). Se obtuvo, entonces, el índice COMP-2 en cada fase narrativa y se aplicó una $t$ de Student para conocer si estas variaciones presentan relevancia estadística, lo que se muestra en la Tabla 4. Cabe recordar que, dado que no todos los niños produjeron el final en sus recontados, los análisis relacionados a dicha fase solo contemplan los niños que lo produjeron ( $\mathrm{n}=11$ para $\mathrm{G} 1 ; \mathrm{n}=18$ para $\mathrm{G} 2$ ).

La Tabla 4 muestra que, si bien en cada fase aumenta la relación entre cláusulas subordinadas y oraciones complejas, este aumento no es significativo al comparar los relatos de niños escolares y preescolares. Esto sugiere que la densidad clausular en cada de categoría se mantiene estable, a pesar de que los niños realizan recontados con un mayor número de subordinaciones y oraciones complejas. 


\begin{tabular}{|l|c|c|c|c|c|c|}
\hline & \multicolumn{2}{|c|}{ Grupo 1 } & \multicolumn{2}{c|}{ Grupo 2 } & & \\
\hline & $\mathrm{x}$ & D.E. & $\mathrm{x}$ & $\mathrm{D} . \mathrm{E}$. & $t(p)$ & $r$ \\
\hline Presentación & 1,03 & 0,45 & 1,10 & 0,17 & $-0,6(, 26)$ & 0,10 \\
\hline Episodios & 1,12 & 0,14 & 1,13 & 0,17 & $-0,3(, 40)$ & 0,04 \\
\hline Final & 1,25 & 0,42 & 1,36 & 0,59 & $-0,5(, 44)$ & 0,03 \\
\hline
\end{tabular}

x: promedio. D.E.: desviación estándar. t: valor de t en el contraste de medias. p: significancia. r: tamaño del efecto.

Tabla 4. Comparación de los promedios grupales para el índice COMP-2 en cada fase de la estructura narrativa

Por último, para explorar si existen diferencias por el tipo de subordinada, se obtuvo el índice COMP-2 para cada una de ellas en cada etapa de los recontados y se aplicó una prueba t de Student para contrastar los grupos. Los resultados se muestran en la Tabla 5 para la presentación, en la Tabla 6 para episodios y en la Tabla 7 para el final.

\begin{tabular}{|c|c|c|c|c|c|c|}
\hline & \multicolumn{2}{|c|}{ Grupo 1} & \multicolumn{2}{|c|}{ Grupo 2} & \multirow[b]{2}{*}{$t(p)$} & \multirow[b]{2}{*}{$r$} \\
\hline & $\begin{array}{c}x \\
\text { COMP-2 }\end{array}$ & $\begin{array}{c}\text { D.E. } \\
\text { COMP-2 }\end{array}$ & $\begin{array}{c}x \\
\text { COMP-2 }\end{array}$ & $\begin{array}{l}\text { D.E. } \\
\text { COMP-2 }\end{array}$ & & \\
\hline $\begin{array}{l}\text { Subordinadas } \\
\text { adjetivas }\end{array}$ & 0,54 & 0,61 & 0,30 & 0,36 & $1,5(, 07)$ & 0,24 \\
\hline $\begin{array}{l}\text { Subordinadas } \\
\text { sustantivas }\end{array}$ & 0,26 & 0,46 & 0,54 & 0,34 & $-2,2(, 002)^{*}$ & 0,34 \\
\hline $\begin{array}{l}\text { Subordinadas } \\
\text { adverbiales }\end{array}$ & 0,19 & 0,37 & 0,24 & 0,37 & $-0,4(, 34)$ & 0,06 \\
\hline \multicolumn{7}{|c|}{$\begin{array}{l}\text { x: promedio. D.E.: desviación estándar. t: valor de t en el contraste de medias. p: significancia. } r \text { : tamañ } \\
\text { del efecto. } \\
* \text { p }<.05\end{array}$} \\
\hline
\end{tabular}

Tabla 5. Comparación de los promedios del índice COMP-2 para cada tipo de subordinada en la fase de presentación.

En la Tabla 5 se observa que en la fase de presentación, los niños de primero básico producen un menor número de subordinadas adjetivas y aumentan la producción de sustantivas y adverbiales. De estas variaciones, solo el incremento de subordinadas sustantivas por oración compleja es significativo.

\begin{tabular}{|l|c|c|c|c|c|c|}
\hline & \multicolumn{2}{|c|}{ Grupo 1 } & \multicolumn{2}{c|}{ Grupo 2 } & & \\
\hline & $\begin{array}{c}\mathrm{x} \\
\text { COMP-2 }\end{array}$ & $\begin{array}{c}\text { D.E. } \\
\text { COMP-2 }\end{array}$ & $\begin{array}{c}\mathrm{x} \\
\text { COMP-2 }\end{array}$ & $\begin{array}{c}\text { D.E. } \\
\text { COMP-2 }\end{array}$ & $t(p)$ & $r$ \\
\hline $\begin{array}{l}\text { Subordinadas } \\
\text { adjetivas }\end{array}$ & 0,49 & 0,77 & 0,45 & 0,83 & $0,13(, 44)$ & 0,02 \\
\hline $\begin{array}{l}\text { Subordinadas } \\
\text { sustantivas }\end{array}$ & 0,40 & 0,26 & 0,47 & 0,27 & $-0,97(, 17)$ & 0,16 \\
\hline $\begin{array}{l}\text { Subordinadas } \\
\text { adverbiales }\end{array}$ & 0,68 & 0,32 & 0,62 & 0,24 & $0,59(, 28)$ & 0,10 \\
\hline
\end{tabular}

x: promedio. D.E.: desviación estándar. t: valor de t en el contraste de medias. p: significancia. r: tamaño del efecto. 
Tabla 6. Comparación de los promedios del índice COMP-2 para cada tipo de subordinada en la fase de episodios.

En la Tabla 6 se observa que la producción de subordinadas en los episodios de niños escolares no se diferencia de la encontrada en los recontados de los niños preescolares, aunque se advierte una disminución en el promedio de adjetivas y adverbiales y un aumento en el de sustantivas.

\begin{tabular}{|l|c|c|c|c|c|c|}
\hline & \multicolumn{2}{|c|}{ Grupo 1 } & \multicolumn{2}{c|}{ Grupo 2 } & & \\
\hline & $\mathrm{x}$ & $\mathrm{D} . \mathrm{E}$. & $\mathrm{x}$ & $\mathrm{D} . \mathrm{E}$. & $\boldsymbol{t}(\boldsymbol{p})$ & $\boldsymbol{r}$ \\
\hline $\begin{array}{l}\text { Subordinadas } \\
\text { adjetivas }\end{array}$ & 0,5 & 0,15 & 0,0 & 0,0 & $1,0(, 09)$ & 0,26 \\
\hline $\begin{array}{l}\text { Subordinadas } \\
\text { sustantivas }\end{array}$ & 0,75 & 0,35 & 1,17 & 0,43 & $-2,6(, 01)^{*}$ & 0,45 \\
\hline $\begin{array}{l}\text { Subordinadas } \\
\text { adverbiales }\end{array}$ & 0,45 & 0,36 & 0,21 & 0,31 & $1,8(, 04)^{*}$ & 0,33 \\
\hline $\begin{array}{l}\text { x: promedio. D.E.: desviación estándar. t: valor de t en el contraste de medias. p: significancia. r: tamaño } \\
\text { del efecto. } \\
* \\
\text { p <.05 }\end{array}$
\end{tabular}

Tabla 7. Comparación de los promedios del índice COMP-2 para cada tipo de subordinada en la fase de final.

Por último, los niños en etapa escolar producen un mayor número de subordinadas sustantivas que los niños de preescolar al recontar la categoría final; en contraste, la proporción de adverbiales disminuye. Ambas variaciones son estadísticamente significativas, aunque con un tamaño del efecto pequeño en el caso de las adverbiales. El número de subordinadas adjetivas, por su parte, es marginal en los recontados del grupo 1 , y ningún niño del grupo 2 que produjo final incluyó adjetivas.

En síntesis, los recontados de los niños de primero básico se caracterizan por presentar un mayor número de subordinadas sustantivas por oración compleja, lo que se manifiesta especialmente en las etapas de presentación y final, y por una disminución de las adverbiales en el final; por el contrario, no se observa que varíe la densidad clausular en el recontado de episodios al comparar las producciones del grupo 1 con el grupo 2.

\section{Discusión}

El objetivo de este trabajo fue observar el desarrollo de la sintaxis compleja en recontados narrativos sin apoyo de láminas entre un grupo de niños pre-escolares y otro de niños que cursan primer año básico. La observación de la complejidad sintáctica se realizó a través de un conjunto de medidas lingüísticas de complejidad y gramaticalidad. Los principales resultados obtenidos indican que:

1. Los recontados de los niños pre-escolares y de primero básico no se diferencian en el número de cláusulas por oración; es decir, presentan una complejidad sintáctica similar. Sin embargo, los niños de primer año sí producen un mayor número de cláusulas subordinadas por oración compleja que los preescolares; esto es, sus oraciones complejas son más densas.

2. En los recontados de los dos grupos, las cláusulas subordinas que más aparecen son las sustantivas y las adverbiales. De estas, solo las sustantivas aumentan significativamente al relacionarlas con el total de oraciones complejas; es decir, el incremento de la densidad clausular global se basa en una mayor producción de subordinadas nominales. 
3. La complejidad sintáctica, medida como densidad clausular, no se modifica significativamente en ninguna de las fases de la secuencia narrativa (presentación, episodios, final) al contrastar a los dos grupos.

4. En general, en las distintas categorías narrativas tampoco se presentan diferencias según el tipo de subordinada al contrastar los dos grupos de edad, excepto por el aumento en la producción de subordinadas sustantivas por oración compleja, tanto en la presentación como en el final, y la disminución de adverbiales en la etapa final.

Este conjunto de resultados indica que nuestra hipótesis inicial se cumplió solo parcialmente, dado que se observó un aumento en la complejidad sintáctica solo en algunos índices medidos. Frente a esto, un primer punto de discusión que surge es en torno al concepto mismo de desarrollo. El desarrollo del lenguaje, como cualquier fenómeno de desarrollo humano, es un proceso de recurrencias y transformaciones, no solo de cambios sucesivos y lineales (Bronfenbrenner, 1979). Probablemente, en algunos estadios, los cambios resulten más evidentes y acelerados; en otros, será predominante la estabilidad y la acumulación de experiencia para propiciar uno nuevo. Al observar los resultados acá expuestos, queda en evidencia que el desarrollo de la sintaxis compleja se puede explicar bajo esta perspectiva. Si bien, los niños más grandes producen un mayor número de oraciones y de cláusulas, el fenómeno de la complejidad sintáctica pareciera cambiar de un grupo a otro en un aspecto principal, la densidad clausular dentro de la oración compleja, y presentar cierta estabilidad en otros, como en la producción de subordinadas adjetivas. Es evidente que no todos los aspectos de un fenómeno tienen que desarrollarse al mismo tiempo o con el mismo ritmo, y que la edad no es el único factor que explica los cambios y recurrencias observados.

Para dar cuenta cabal del desarrollo, se debería complejizar el método de identificación de los fenómenos observados. Estudios como este suelen contabilizar, por ejemplo, una subordinada adverbial causal cuando su producción es gramatical y con un significado coherente. Los fenómenos así descritos son dicotómicos (presencia / ausencia); sin embargo, un aspecto importante del desarrollo queda no descrito. En los recontados de estos niños abundan las oraciones complejas fallidas, reformuladas con éxito o abandonadas, el uso de subordinantes con valores ambiguos y la emisión incompleta de ciertas estructuras que sí alcanzan claridad en su significado (por ejemplo, las adverbiales comparativas con la forma tan... que..., que suelen aparecer sin la primera parte tan...), todos fenómenos que habría que considerar de alguna manera en la perspectiva del desarrollo. En nuestro ámbito, existe aún una dificultad metodológica para describir un proceso de desarrollo con sus etapas de exploración y de consolidación definitiva, lo que resulta extensivo no solo al desarrollo sintáctico, sino igualmente a otros planos del lenguaje (Torres et al., 2016).

Un aspecto que se ha señalado reiteradamente es la importancia del contexto discursivo para interpretar los datos de desarrollo. Se ha propuesto que el discurso narrativo es un buen medio para observar la complejidad sintáctica en niños (Pavez et al., 2015) y su recorrido de desarrollo, tanto en niños en etapa preescolar (Castilla \& Ericks, 2011) como en etapa escolar (Coloma, Peñaloza \& Fernández, 2007; Peñaloza, 2008). Los resultados de nuestra investigación corroboran dicha apreciación. Al contrastar las producciones de ambos grupos en estudio, hubo aspectos que resultaron similares en las distintas fases de los recontados, y otros que presentaron diferencias, como el aumento de subordinadas sustantivas. Si se observan los resultados a la luz del desarrollo narrativo, se puede observar que estos resultados son coherentes con las etapas que los niños están experimentando en su producción discursiva. Hacia los seis años, los niños han consolidado recontados estructurados, con presentación y episodios completos, y se preparan para incorporar de manera definitiva la fase de final, lo que solo lo logrará la mayoría de niños unos años más tarde (Pavez et al., 2008) Es decir, los recontados de niños de las edades estudiadas presentan una relativa estabilidad discursiva, luego de alcanzar la etapa de estructuración básica sobre los cuatro años de edad, y antes de terminar el proceso después de los seis años. 
Estos resultados pueden interpretarse, entonces, a la luz del desarrollo narrativo señalado. Dada la necesaria interacción que ocurren entre los distintos planos lingüísticos (Serra et al., 2000), es posible entender que la producción de recontados estructurados exige una producción estable de ciertas estructuras complejas, como las subordinadas adjetivas en la presentación o las adverbiales en los episodios, y que, a su vez, el afianzamiento de la producción de estas estructuras complejas permite estructurar relatos de una manera que un niño más pequeño aún no logra realizar.

La diferencia en el uso de un tipo u otro de subordinadas en cada categoría de la secuencia narrativa se ha explicado por motivos funcionales discursivos, en el sentido de que cada categoría plasma distintos significados que movilizan ciertos recursos sintácticos afines y no otros de manera prototípica (Meneses et al., 2012). Los resultados de esta investigación parecen señalar que los niños ya han estabilizado una manera de plasmar sintácticamente los significados discursivos propios de los episodios, pero que aún están por estabilizarse las estrategias en torno a las categorías de presentación y final. El hecho de que al comparar los dos grupos no aumente la complejidad global, pero sí la densidad clausular dentro de las oraciones complejas, y que las diferencias no se observen en todas las subordinadas y solo en ciertas categorías narrativas puede dar cuenta de ajustes y precisiones en torno a los fines retóricos de la actividad lingüística. En particular, el importante cambio observado en las oraciones sustantivas entre un grupo y otro puede deberse a un aumento en los discursos directos emitidos y en el uso de infinitivos no clausulares, asociado a una búsqueda de mayor adecuación pragmática del relato (Auza \& Alarcón, 2011), aunque habría que realizar un estudio específico al respecto para obtener información más concluyente.

En el estudio ya mencionado con niños colombianos (Castilla \& Eriks, 2011), las medidas globales de complejidad resultaron ser sensibles para distinguir la producción de niños de distintas edades, lo que no ocurre del mismo modo en los recontados acá observados, pues muchos índices no diferenciaron la producción de ambos grupos. Esto puede indicar que, más allá de considerar el discurso narrativo como un método idóneo para estudiar estos temas, habría que profundizar en la influencia que ejercen los distintos métodos de obtención de las muestras. En niños mayores se ha observado que los rendimientos sintáctico-discursivos en oralidad y escritura son distintos (Meneses et al., 2012); sin embargo, hasta donde se posee información, en el contexto hispanoamericano no se ha contrastado con una misma muestra de niños la producción a partir de recontados con y sin apoyo de láminas, incluso para un mismo guion o para dos guiones de una misma historia. Solo como hipótesis inicial, podría pensarse que recontados con apoyo de láminas facilitan la producción de un mayor número de oraciones, con mayor presencia de yuxtaposición y coordinación de cláusulas; en contraste, el recuento sin apoyo de imágenes debería propiciar recontados más breves y con información más condensada. Muy probablemente, la forma en que están estructurados los relatos también influya en una mayor o menos producción de estructuras complejas. Por ejemplo, en los recontados utilizados son muy relevantes las cualidades de los personajes (plasmados en subordinadas adjetivas) y sus intenciones (plasmadas en adverbiales causales y finales).

Un constante factor de cuidado a la hora de proyectar conclusiones en este tipo de investigaciones es la conformación de las muestras, dado que el número de participantes es pequeño y los criterios de muestreo y selección pueden incorporar sesgos importantes. Los resultados de este estudio solo pueden ser tomados como tendencias en relación a la población que representan. Queda por explorar, además, cómo se modifican las tendencias acá expuestas en niños con otras características socioambientales, incluso dentro de lo que se considere desarrollo típico.

No obstante lo anterior, resultados como este permiten enriquecer el conocimiento con que se cuenta actualmente sobre el desarrollo sintáctico-discursivo de niños hispanohablantes, además de complejizar la reflexión teórica sobre el fenómeno del desarrollo y proyectar algún uso hacia áreas afines de conocimiento, como lo es la identificación de niños con TEL. Las medidas acá descritas han sido utilizadas en diversas investigaciones que buscan marcadores lingüísticos en lenguaje espontáneo para diferenciar niños con y sin trastorno (Restrepo, 
1998; Simon-Cerejeido \& Gutiérrez-Clellen, 2007; Jackson-Maldonado, 2011). Más allá de la identificación, el conocimiento sobre el desarrollo de la complejidad sintáctica en niños típicos podría permitir entender de mejor manera el mismo desarrollo en niños con dificultades del lenguaje, especialmente para valorar si algunos fenómenos resultan particulares o compartidos entre los grupos. Existe, de hecho, la certeza de que el discurso narrativo permite que tanto los niños sin problemas como con TEL eliciten enunciados con mayor complejidad sintáctica (Gutiérrez-Clellen y Hofstetter, 1994; Wagner et al, 2000). En ese sentido, los resultados acá expuestos vuelven a poner sobre la palestra la sensibilidad de estos marcadores frente a la edad, la estrategia general de elicitación de lenguaje (narración versus conversación, por ejemplo), y los métodos específicos que se escojan, como es el recontado sin apoyo de imágenes.

\section{Referencias}

Alarcón, L., \& Auza, A. (2015). Uso y función de nexos en la subordinación y en la coordinación. Evidencia de dos tareas narrativas de niños mexicanos de primero de primaria. En E. Velázquez \& I. Rodríguez (Eds.), Estudios de Lingüística Funcional, (pp. 223-251). Querétaro: Editorial Universitaria UAQ.

American Speech-Language-Hearing Association (2005). Audiology Information Series [en línea]. Disponible en: http://www.asha.org/uploadedFiles/AIS-Hearing-Loss-Types-DegreeConfiguration.pdf\#search=\%22Hearing\%22 (consultado en abril 2017)

Auza, A., \& Alarcón, L. (2011). Cláusulas subordinadas y coordinadas en dos tareas narrativas producidas por niños mexicanos de primero de primaria. En A. M. Cestero, I. Molina, \& F. Paredes (Eds.), Documentos para el XVI Congreso Internacional de la ALFAL Alcalá de Henares 6-9 de junio de 2011. Universidad de Alcalá.

Bedore, L. M., Pena, E. D., Gillam, R. B., \& Ho, T. H. (2010). Language sample measures and language ability in Spanish-English bilingual kindergarteners. Journal of communication disorders 43(6), 498-510.

Benítez, R. \& Alvarado, C. (2013). Un análisis descriptivo de la arquitectura sintáctica de la oralidad. Foro Educacional 22, 13-30.

Bishop, D. V. M. \& Adams, C. (1990). A prospective study of the relationship between specific language impairment, phonological disorders and reading retardation. Journal of Child Psychology and Psychiatry and Allied Disciplines 31, 1027-1050.

Bronfenbrenner, U. (1979). La ecología del desarrollo humano. Barcelona: Paidós.

Brown, R. (1973). A First Language: The Early Stages. Cambridge: Harvard University Press.

Castilla, A., \& Eriks, A. (2011). Medidas de evaluación de lenguaje espontáneo en niños hispanohablantes en edad preescolar. Revista de Logopedia, Foniatría y Audiología 32, e7-e19.

Checa, I. (2003). Madurez sintáctica y subordinación: los índices secundarios clausales. Interlingüística 14, 191-206.

Coloma, C. J., Peñaloza, C. \& Fernández, R. (2007). Producción de oraciones complejas en niños de 8 y 10 años. Revista de Lingüística Teórica y Aplicada 45(1), 33-44. https://doi.org/10.4067/ S0718-48832007000100003

Crespo, N., Alfaro, P. \& Góngora, B. (2011). La medición de la sintaxis: evolución de un concepto. Onomázein 24, 155-172. 
Gili Gaya, S. (1948). Curso superior de sintaxis española. Barcelona: Bibliograf.

Gili Gaya, S. (1972). Estudios de lenguaje infantil. Barcelona: Bibliograf.

Gutiérrez-Clellen, V., \& Hofstetter, R. (1994). Syntactic complexity in Spanish narratives: a developmental study. Journal of Speech \& Hearing Research 37(3), 645-654.

Hunt, K. (1965). Grammatical structure written at three grade levels. National Council of Teachers of English Research, Report $\mathrm{N}^{\circ} 3$.

Hunt, K. (1970). Recent measures in syntactic development. In Readings in Applied Trasformational Grammar (pp. 187-200).

Jackson, D. \& Maldonado, R. (2015). La complejidad sintáctica en niños pre-escolares y escolares. Con y sin trastorno primario del lenguaje. En E. Velásquez \& I. Rodríguez (Eds.), Estudios de Lingüística Funcional (pp. 253-301). Querétaro: Editorial Universitaria UAQ.

Loban, W. (1970). Stages, Velocity, and Prediction of Langauge Development: Kindergarten through Grade Twelve. Final Report.

McCarthy, D. (1954). Language development in children. En L. Carmichael (Ed.), Manual of Child Psychology. New York: John Wiley \& Sons Inc.

Meneses, A., Ow, M. \& Benítez, R. (2012). Complejidad sintáctica: ¿modalidad comunicativa o tipo textual? Estudio de casos de producciones textuales de estudiantes de $5^{\circ}$ básico. Onomázein $25,65-93$.

Muñoz, G., Echeverría, M. \& Véliz, M. (1985). madurez sintáctica y combinación de oraciones en estudiantes universitarios. Revista de Lingüística Teórica y Aplicada 23, 107-120.

Pavez, M. M., Coloma, C. J., Araya, C., Maggiolo, M. \& Peñaloza, C. (2015). Gramaticalidad y complejidad en narración y conversación en niños con trastorno específico del lenguaje. Revista de Logopedia, Foniatría y Audiología 35, 150-158. https://doi.org/10.1016/j.rlfa.2015.07.004

Pavez, M. M., Coloma, C. J. \& Maggiolo, M. (2008). El desarrollo narrativo en niños. Una propuesta práctica para la evaluación y la intervención en niños con trastorno del lenguaje. Barcelona: Ars Médica.

Peñaloza, C. (2008). Producción de oraciones complejas por niños de 6, 8 y 10 años en la actividad discursiva de recontado narrativo [Tesis de magister]. Pontificia Universidad Católica de Chile.

Raven, J. (2005). Test de Matrices Progresivas. Escala Coloreada, General y Avanzada. Buenos Aires: Paidós.

Real Academia Española. (2010). Nueva gramática de la lengua española. Ciudad de México: Editorial Planeta Mexicana.

Restrepo, M. A. (1998). Identifiers of predominantly Spanish-Speaking children with language impairment. Journal of Speech, Language, and Hearing Research 41, 1398-1411.

Rojas, C. (2009). Starting small: Effects in the acquisition of early relative constructions in Spanish. In 12th Rice University Symposium on Language: The Genesis of Syntactic Complexity.

Serra, M., Serrat, E., Solé, R., Bel, A. \& Aparici, M. (2000). La adquisición del lenguaje. Barcelona: Ariel. 
Shum, G. (1993). El pronombre en el lenguaje formal del niño. Infancia y Aprendizaje 61, 107121.

Silva, M. L. \& Plana, M. D. (2014). Estrategias de relativización en niños pequeños: comparación de desempeños ante producción espontánea y recuperación de relatos. Logos: Revista de Lingüística, Filosofía y Literatura 24(2), 101-122.

Simon-Cereijido, G. \& Gutiérrez-Clellen, V. (2007). Spontaneous language markers of Spanish language impairment. Applied Psycholinguistics 28, 317-339.

Storch, S. \& Whitehurst, G. (2002). Oral Language and Code-Related Precursors to Reading: Evidence From a Longitudinal Structural Model. Developmental Psychology 38 (6), 934-947

Thordardottir, E. (2008). Language specific effects of task demandson the manifestation of specific language impairment: A comparison of English and Icelandic. Journal of Speech, Language, and Hearing Research 51, 922-937.

Torres, F.; Pino, J.; Peñaloza, C.; Rojas, D.; Fernández, M. A.; Castro, P.; Bustos, M. \& Hormazábal, K. (2016). Comparación de criterios para determinar la edad de adquisición de fonemas lateral y róticos. Revista Chilena de Fonoaudiología 15, 1-16.

Véliz, M. (1988). Evaluación de la madurez sintáctica en el discurso. Revista de Lingüística Teórica \& Aplicada 26, 105-141.

Wagner, C.; Nettelbladt, U.; Sahlen, B., \& Nilholm, C. (2000). Conversation versus narration in pre-school children with language impairment. International Journal of Language and Communication Disorders, 35, 83-93.

Notas

1. Agradecemos la ayuda recibida a través del Proyecto Concurso en Ciencias Sociales, Humanidades y Educación VID 2009 Desempeño lingüístico, lector, cognitivo y auditivo en alumnos de $1^{\circ}$ básico con problemas de lenguaje que asisten a escuelas con proyectos de integración, financiado por la Universidad de Chile, con referencia SOC09/03-2. 